\title{
USDA
}

United States Department of Agriculture

\section{Grain Truck and Ocean Rate Advisory}

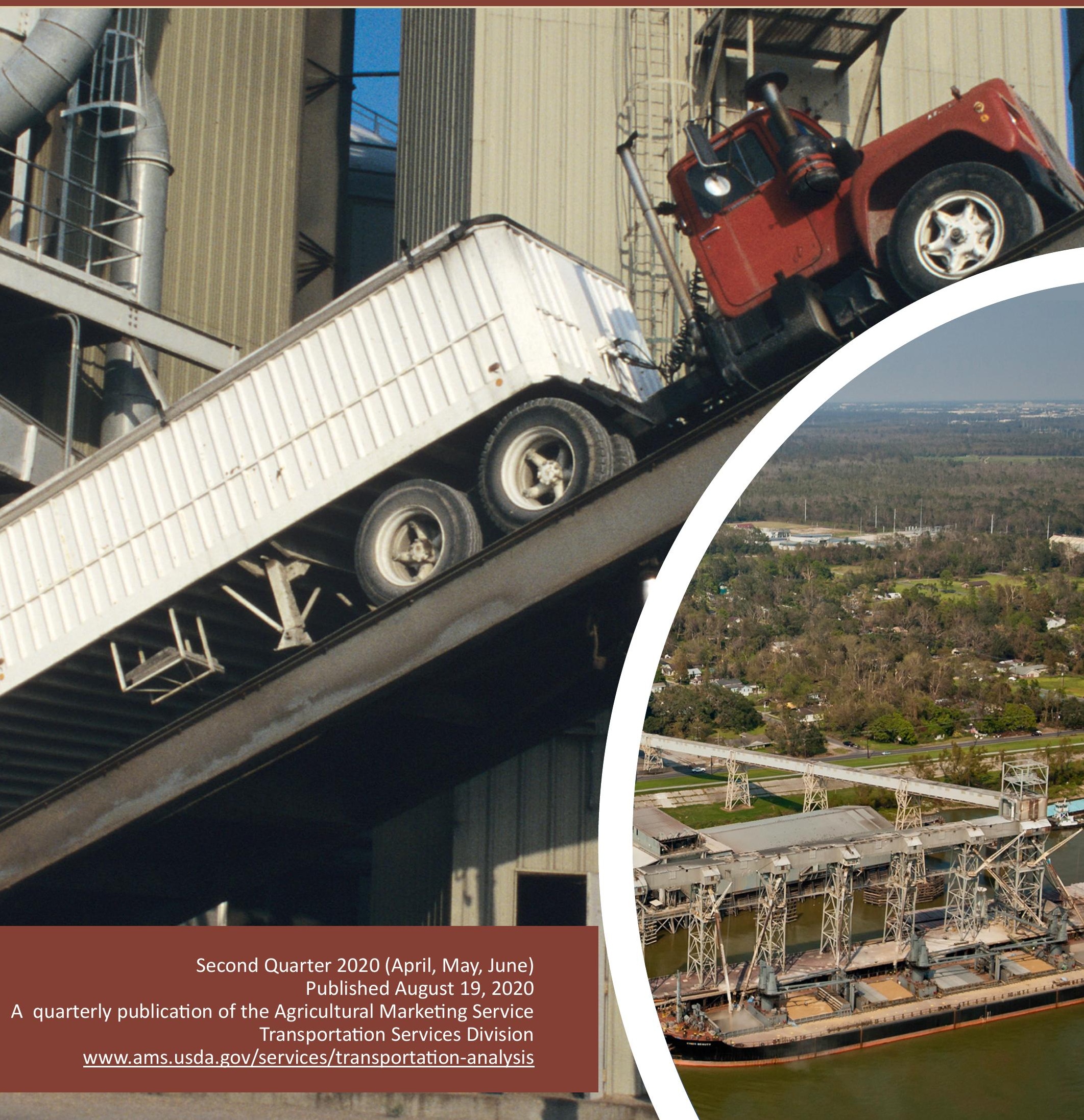




\section{Grain Truck and Ocean Rate Advisory}

\section{TRUCK ADVISORY}

Truck advisory presents overall situation of grain truck transportation market including national and regional truck rates, truck availability, truck usage and diesel fuel prices.

Table 1. U.S. grain truck market, 2nd quarter 2020

\begin{tabular}{|c|c|c|c|c|c|c|}
\hline & 25 miles & 100 miles & 200 miles & Truck availability & Truck use & Future truck use \\
\hline & \multirow{2}{*}{\multicolumn{3}{|c|}{${ }^{1}$ Rate per mile, per truckload }} & \multicolumn{3}{|c|}{ Quarterly index* } \\
\hline & & & & $\begin{array}{c}1 \text { = Very easy } \\
\text { to } \\
5 \text { = Very difficult }\end{array}$ & \multicolumn{2}{|c|}{$\begin{array}{c}1 \text { = Much lower } \\
\text { to } \\
5 \text { = Much higher }\end{array}$} \\
\hline National average $^{2}$ & 4.14 & 2.50 & 2.09 & 2.50 & 3.50 & 3.36 \\
\hline North Central & 3.82 & 2.42 & 2.00 & 2.50 & 3.50 & 3.38 \\
\hline East & 3.56 & NA & NA & 1.50 & 4.00 & 4.00 \\
\hline South Central & 3.20 & 2.97 & 2.30 & 4.00 & 3.00 & 3.00 \\
\hline West & 3.72 & NA & NA & 2.00 & 3.00 & 3.00 \\
\hline Rocky Mountain & 5.16 & NA & NA & 3.00 & 3.50 & 3.00 \\
\hline
\end{tabular}

${ }^{1}$ Rates are based on trucks with 80,000-pound (lb) gross vehicle weight limit, and are quoted in U.S. dollars

${ }^{2}$ National average is based on rates received from various States, but not every State is represented.

*Current and future truck use indices are based on comparison to the same quarter last year.

Note: NA = not available because of low or no response rate.

Source: USDA, Agricultural Marketing Service.

Figure 1. U.S. Grain Truck Market, 2nd quarter 2020

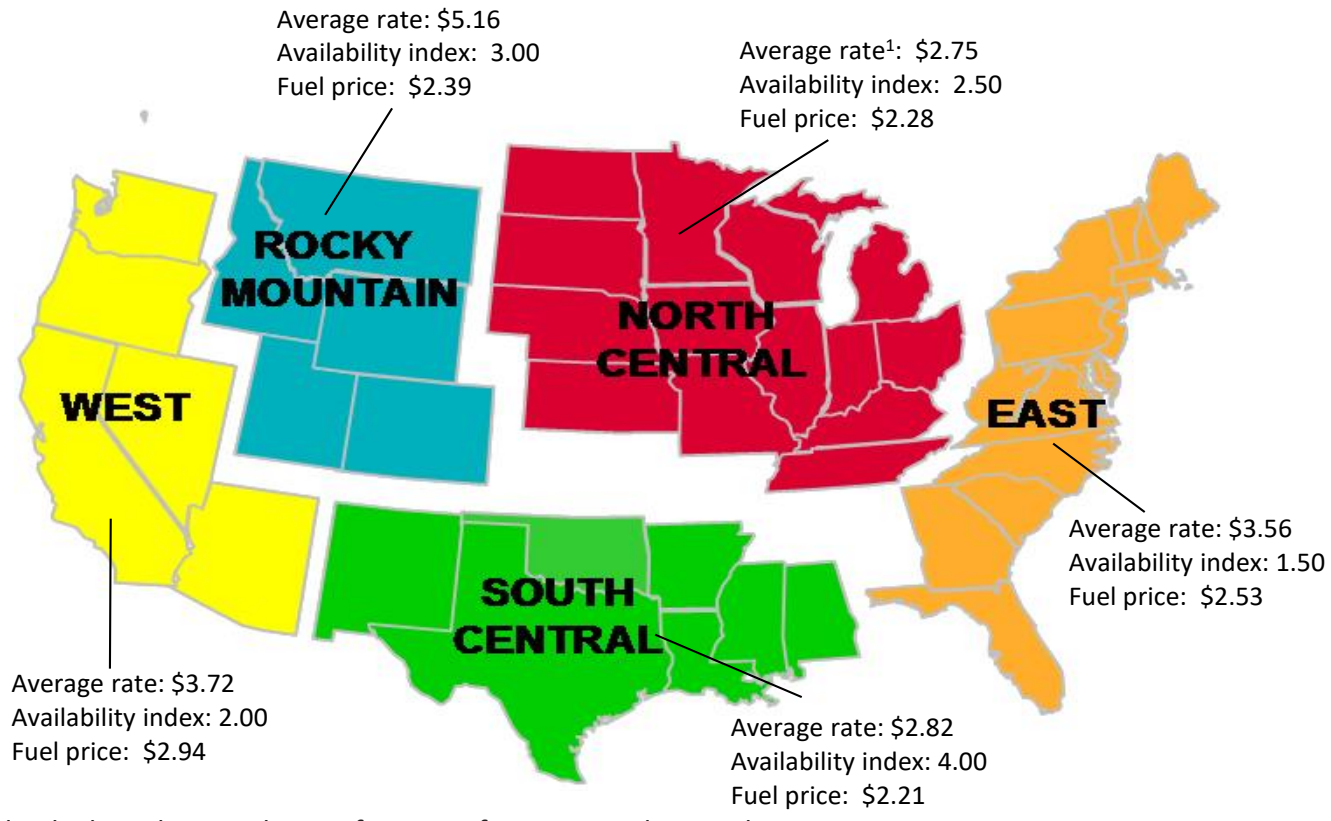

${ }^{1}$ Average rate per loaded mile, based on truck rates for trips of 25, 100, and 200 miles.

Note: Fuel prices are a quarterly average (unit per gallon).

Source: Fuel price data are from U.S. Department of Energy, Energy Information Administration, and availability index data are from USDA,

Agricultural Marketing Service. 


\section{Grain Truck and Ocean Rate Advisory}

\section{TRUCK USE}

Truck use indices represent current and future national and regional truck use.

Table 2. Regional truck use index*

\begin{tabular}{|c|c|c|c|c|c|c|c|c|}
\hline \multicolumn{5}{|c|}{$\begin{array}{c}\text { Current truck use } \\
1=\text { Much lower to } 5=\text { Much higher }\end{array}$} & \multicolumn{4}{|c|}{$\begin{array}{c}\text { Future truck use } \\
1=\text { Much lower to } 5=\text { Much higher }\end{array}$} \\
\hline 2019 & 1st qtr. & 2nd qtr. & $3 r d$ qtr. & 4th qtr. & 1st qtr. & 2nd qtr. & 3rd qtr. & 4th qtr. \\
\hline National & 3.40 & 3.04 & 3.45 & 3.40 & 3.60 & 3.41 & 2.92 & 3.30 \\
\hline North Central & 3.20 & 2.88 & 3.57 & 3.40 & 3.30 & 3.53 & 2.33 & 3.30 \\
\hline East & NA & 3.67 & NA & NA & NA & 2.67 & NA & NA \\
\hline South Central & 3.80 & 3.25 & 3.50 & 3.50 & 4.00 & 3.50 & 2.33 & 3.50 \\
\hline West & NA & 3.00 & 3.00 & 3.00 & NA & 3.50 & 3.50 & 3.00 \\
\hline Rocky Mountain & NA & NA & NA & NA & NA & NA & NA & NA \\
\hline 2020 & 1st qtr. & 2nd qtr. & $3 r d$ qtr. & 4th qtr. & 1st. qtr. & 2nd qtr. & 3rd qtr. & 4th qtr. \\
\hline National & 3.20 & 3.50 & & & 2.80 & 3.36 & & \\
\hline North Central & 3.30 & 3.50 & & & 2.80 & 3.38 & & \\
\hline East & NA & NA & & & NA & NA & & \\
\hline South Central & 3.00 & 3.00 & & & 3.00 & 3.00 & & \\
\hline West & 3.00 & 3.00 & & & 2.50 & 3.00 & & \\
\hline Rocky Mountain & NA & 3.50 & & & NA & 3.00 & & \\
\hline
\end{tabular}

*Current and future truck use indices are based on comparison to the same quarter last year. Note: qtr. = quarter; NA = not available.

Source: USDA, Agricultural Marketing Service.

Figure 2. National truck usage, 2nd quarter 2020

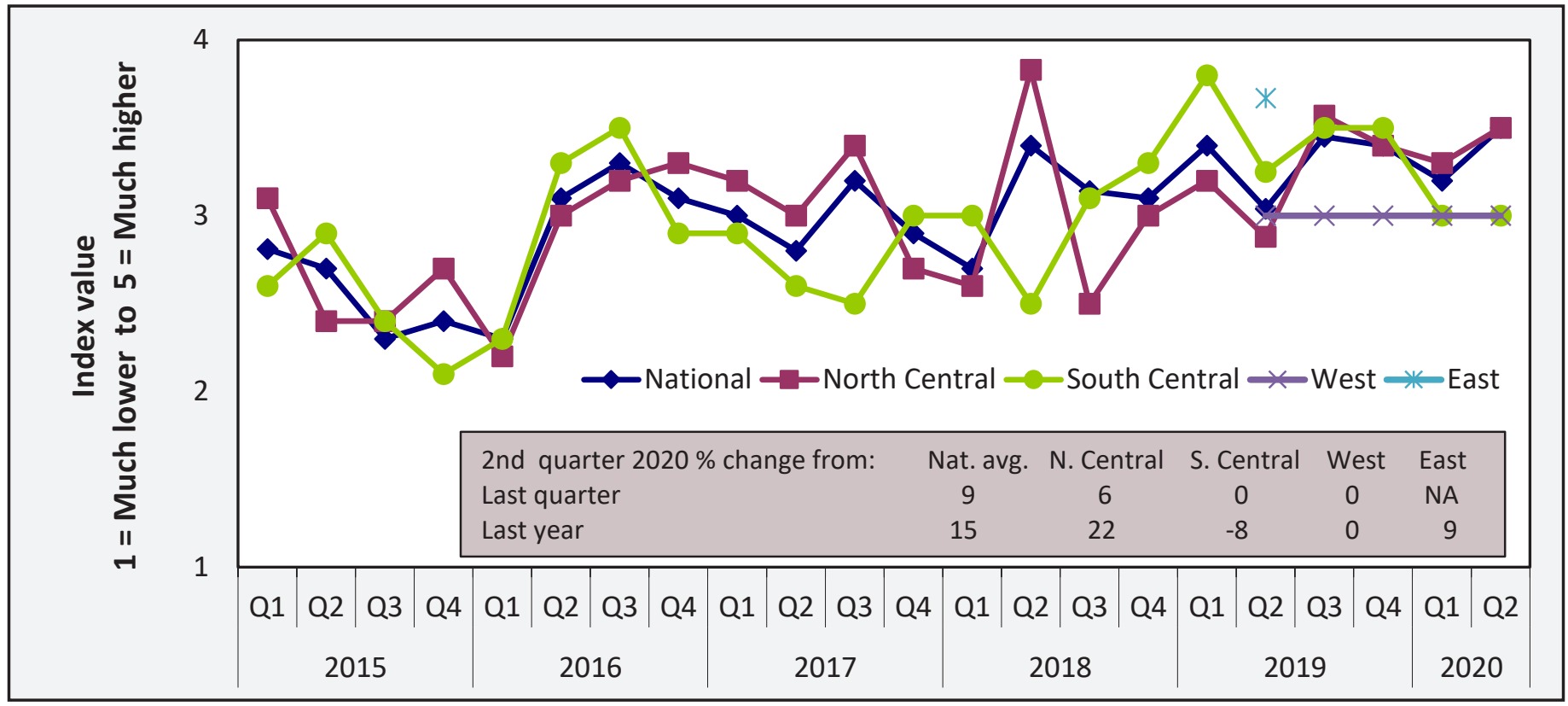

Note: $\mathrm{Q}=$ quarter; Nat. = national; avg. = average; N. = north; $\mathrm{S} .=$ south; $\mathrm{NA}=$ not available.

Source: USDA, Agricultural Marketing Service. 


\section{Grain Truck and Ocean Rate Advisory}

\section{TRUCK AVAILABILITY}

The truck availability index tracks the trends in perceived ease of hiring a truck as reported by grain elevators.

Table 3. Quarterly national truck availability index

\begin{tabular}{|l|c|c|c|c|c|}
\hline \multirow{2}{*}{ Region } & \multicolumn{2}{|c|}{1 Very easy $\mathbf{5 = \text { Very difficult }}$} & \multicolumn{2}{c|}{ Current quarter as \% change from } \\
\hline & $\begin{array}{c}\text { 2nd qtr. } \\
\mathbf{2 0 2 0}\end{array}$ & Previous qtr. & $\begin{array}{c}\text { Same qtr. } \\
\text { last year }\end{array}$ & Previous qtr. & $\begin{array}{c}\text { Same qtr. } \\
\text { last year }\end{array}$ \\
\hline National & $\mathbf{2 . 5 0}$ & $\mathbf{2 . 5 0}$ & $\mathbf{3 . 1 9}$ & $\mathbf{0}$ & $-\mathbf{2 2}$ \\
\hline North Central & 2.50 & 2.70 & 3.12 & -7 & -20 \\
\hline East & 1.50 & NA & 2.67 & NA & -44 \\
\hline South Central & 4.00 & 1.70 & 4.00 & 135 & 0 \\
\hline West & 2.00 & 3.50 & 3.00 & -43 & -33 \\
\hline
\end{tabular}

Note: qtr. = quarter; $\mathrm{NA}=$ not available.

Source: USDA, Agricultural Marketing Service.

Figure 3. National truck availability

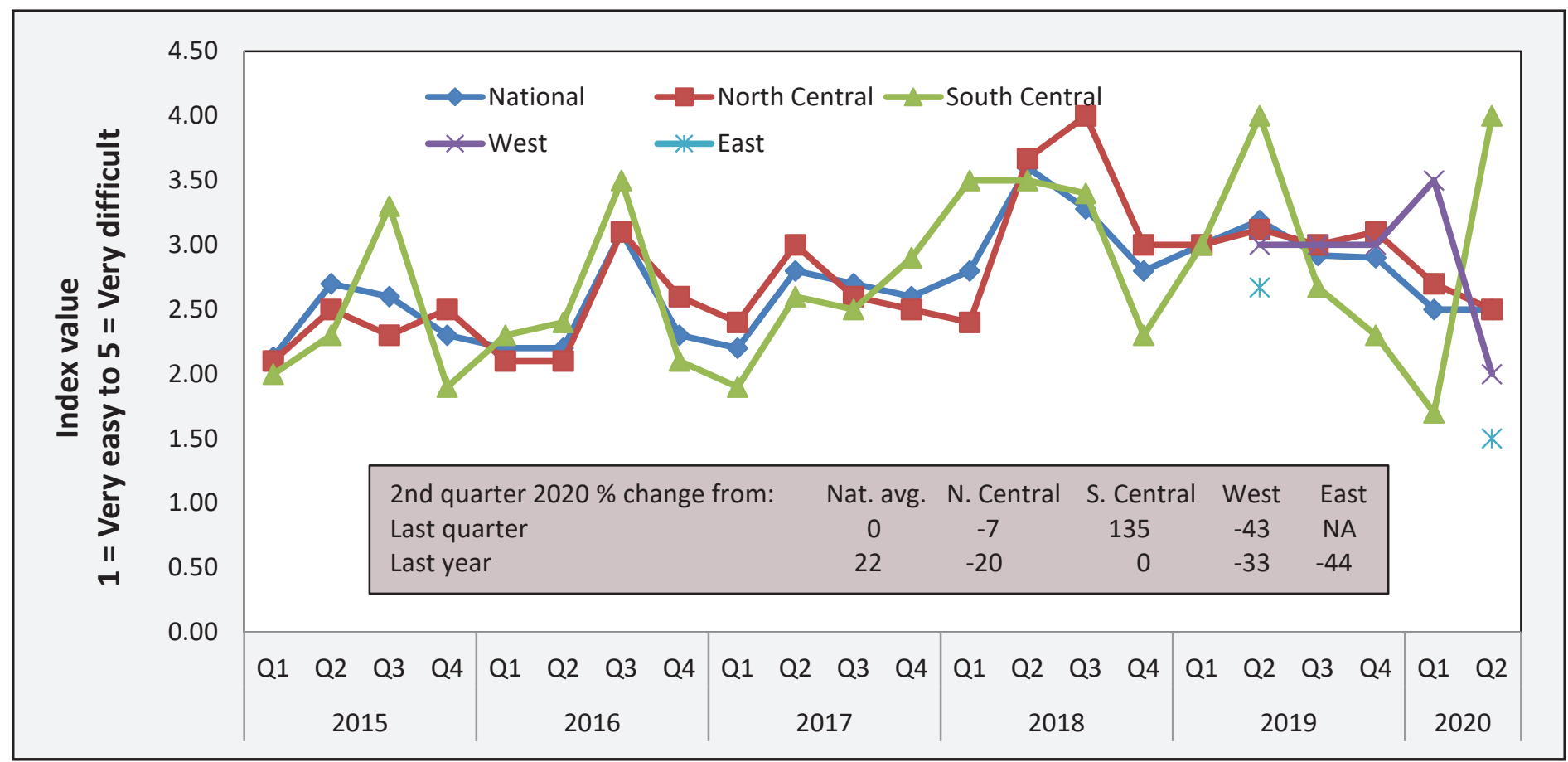

Note: $\mathrm{Q}=$ quarter; Nat. = national; avg. = average; $\mathrm{N}$. = north; $\mathrm{S} .=$ south; $\mathrm{NA}=$ not available.

Source: USDA, Agricultural Marketing Service. 


\section{Grain Truck and Ocean Rate Advisory}

\section{TRUCK RATES}

The truck is assumed to carry 55,000 lbs. or $\mathbf{2 5}$ metric tons of grain. Rates per metric ton per mile can be calculated from rates per truckload.

Table 4. Average grain truck rates for short and long hauls, 2nd quarter 2020

\begin{tabular}{|c|c|c|c|c|c|c|c|c|c|}
\hline \multirow{3}{*}{ Region } & \multirow{2}{*}{\multicolumn{3}{|c|}{ (\$/mile per truckload) }} & \multicolumn{6}{|c|}{$\%$ change from } \\
\hline & & & & \multicolumn{3}{|c|}{ Last qtr. } & \multicolumn{3}{|c|}{ Same qtr. last year } \\
\hline & 25 miles & 100 miles & 200 miles & 25 miles & 100 miles & 200 miles & 25 miles & 100 miles & 200 miles \\
\hline National average & 4.14 & 2.50 & 2.09 & $-9.2 \%$ & $-20.4 \%$ & $-23.4 \%$ & $-1.4 \%$ & $-10.7 \%$ & $-18.4 \%$ \\
\hline North Central & 3.82 & 2.42 & 2.00 & $-17.1 \%$ & $-9.4 \%$ & $-32.0 \%$ & $-12.6 \%$ & $-11.7 \%$ & $-24.0 \%$ \\
\hline East & 3.56 & NA & NA & NA & NA & NA & NA & NA & NA \\
\hline South Central & 3.20 & 2.97 & 2.30 & $0.0 \%$ & $0.0 \%$ & $0.0 \%$ & $-10.1 \%$ & $15.1 \%$ & $13.9 \%$ \\
\hline West & 3.72 & NA & NA & NA & NA & NA & NA & NA & NA \\
\hline Rocky Mountain & 5.16 & NA & NA & NA & NA & NA & NA & NA & NA \\
\hline
\end{tabular}

Note: qtr. = quarter; NA = not available.

Rates are based on trucks with 80,000-pound (Ib) gross vehicle weight limit.

Source: USDA, Agricultural Marketing Service.

Figure 4. National average truck rates by trip distance

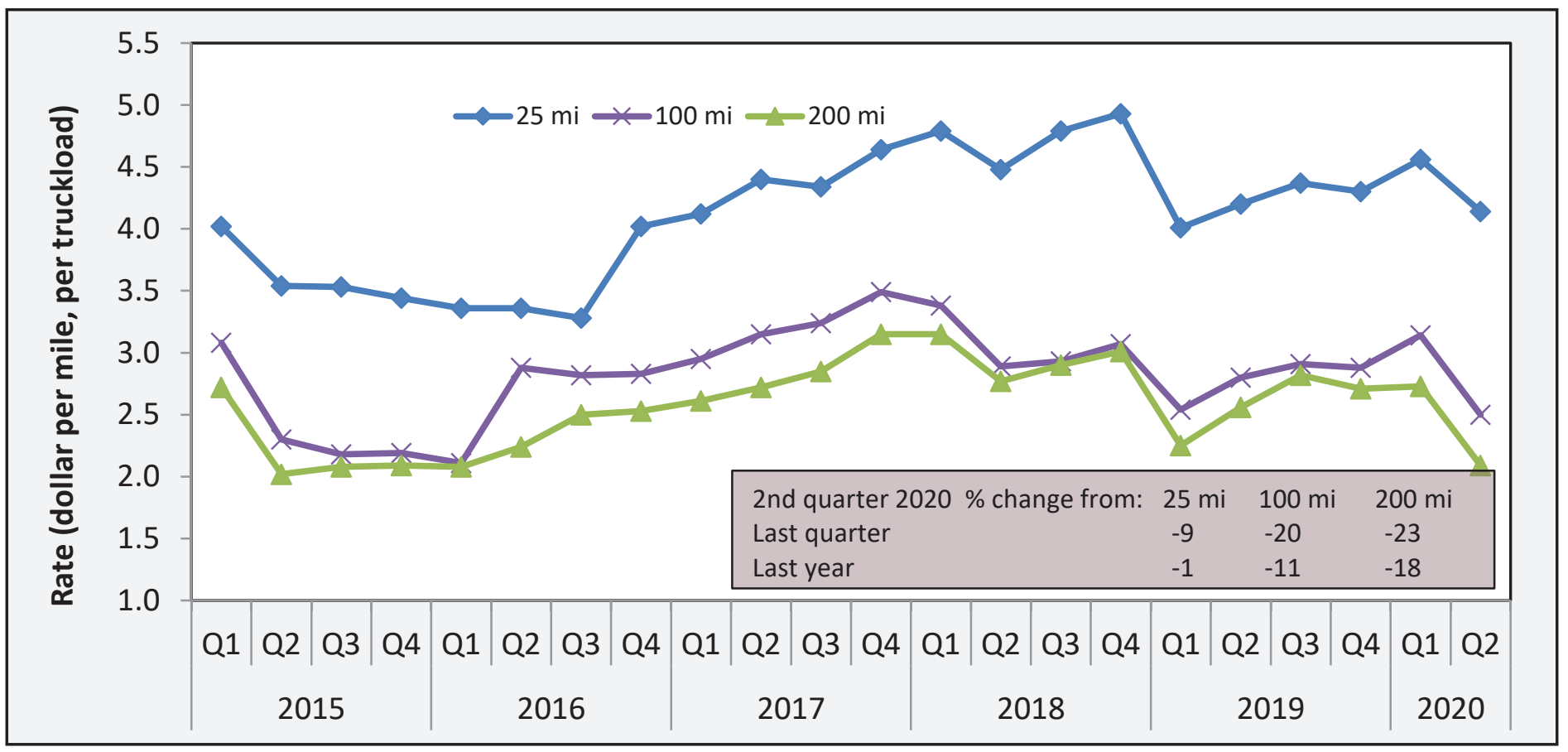

Note: $\mathrm{Q}=$ quarter $; \mathrm{mi}=$ miles .

Source: USDA, Agricultural Marketing Service. 


\section{Grain Truck and Ocean Rate Advisory}

\section{U.S. DIESEL FUEL RATES}

The diesel fuel price provides a proxy for trends in U.S. truck rates. Diesel fuel is a significant expense for grain movements.

Table 5. 2 nd quarter 2020 average diesel fuel prices (all types - \$/gallon)

\begin{tabular}{|c|c|c|c|}
\hline \multirow{2}{*}{ Location } & \multirow{2}{*}{ Price } & \multicolumn{2}{c|}{ Change from } \\
\cline { 3 - 4 } & & Last qtr. & Same qtr. last year \\
\cline { 3 - 4 } & & -0.40 & -0.62 \\
\hline East Coast & 2.53 & -0.37 & -0.55 \\
\hline New England & 2.66 & -0.41 & -0.63 \\
\hline Central Atlantic & 2.71 & -0.41 & -0.63 \\
\hline Lower Atlantic & 2.38 & -0.48 & -0.74 \\
\hline Midwest & 2.28 & -0.44 & -0.68 \\
\hline Gulf Coast & 2.21 & -0.48 & -0.73 \\
\hline Rocky Mountain & 2.39 & -0.50 & -0.76 \\
\hline West Coast & 2.94 & -0.52 & -0.81 \\
\hline California & 3.23 & -0.45 & -0.69 \\
\hline U.S. & 2.43 & & \\
\hline
\end{tabular}

Note: qtr. = quarter.

Source: U.S. Department of Energy, Energy Information Administration.

Figure 5. U.S. average on-highway diesel fuel prices

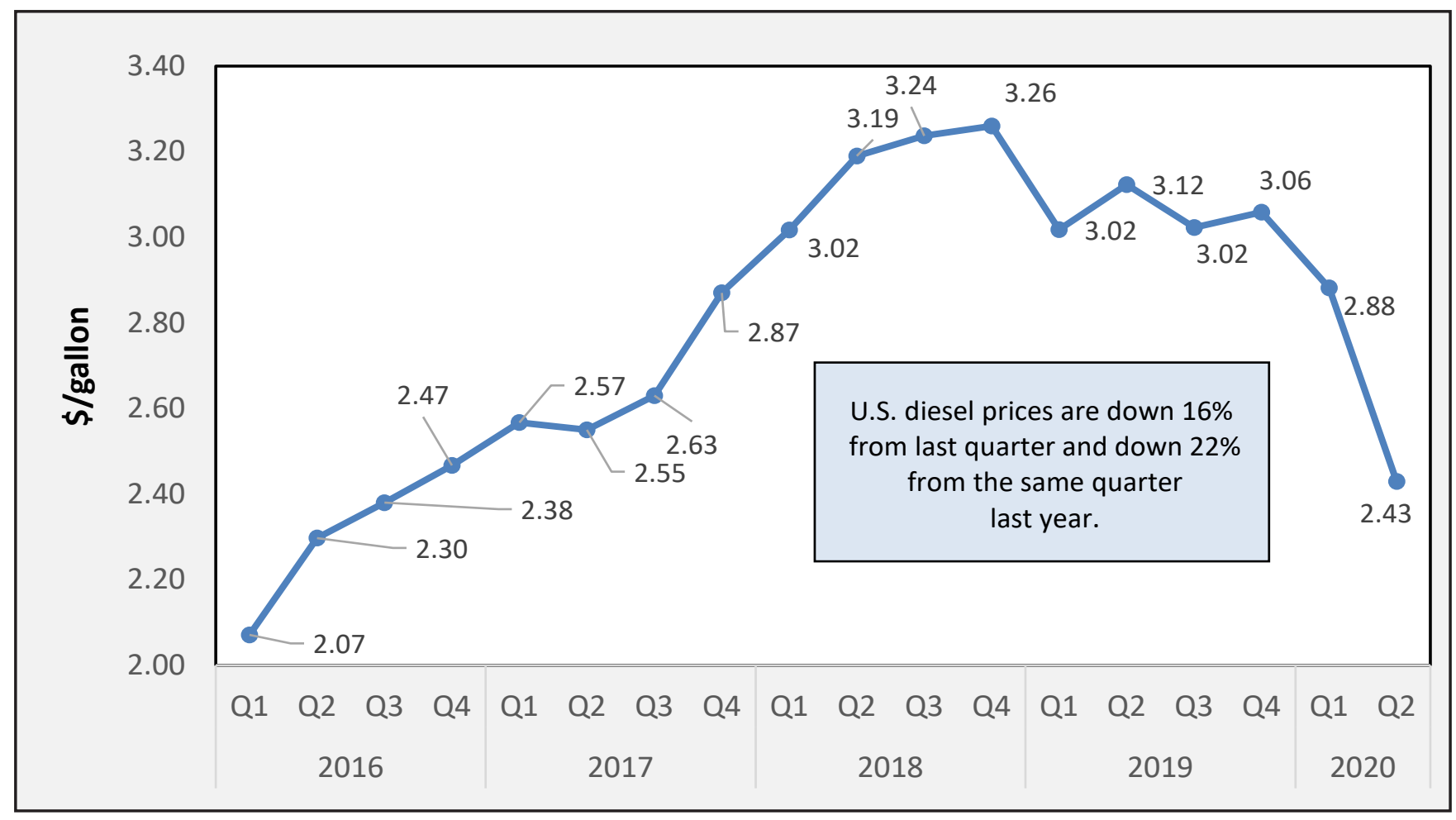

Note: $\mathrm{Q}=$ quarter.

Source: U.S. Department of Energy, Energy Information Administration. 


\section{Grain Truck and Ocean Rate Advisory}

\section{OCEAN RATES}

Quarterly ocean freight rates for shipping bulk grain from the U.S. Gulf and Pacific Northwest to selected foreign markets in dollars per metric ton.

Table 6. Ocean shipping rates for bulk grain (\$/metric ton)

\begin{tabular}{|c|c|c|c|c|c|c|c|c|c|c|}
\hline \multicolumn{11}{|c|}{ U.S. Gulf to } \\
\hline Country & $\begin{array}{c}\text { 1st qtr. } \\
2019\end{array}$ & $\begin{array}{l}\text { 2nd qtr. } \\
2019\end{array}$ & $\begin{array}{c}3 \text { 3rd qtr. } \\
2019\end{array}$ & $\begin{array}{c}\text { 4th qtr. } \\
2019\end{array}$ & Avg. & $\begin{array}{c}\text { 1st qtr. } \\
2020\end{array}$ & $\begin{array}{c}\text { 2nd qtr. } \\
2020\end{array}$ & $\begin{array}{c}3 \text { 3rd qtr. } \\
2020\end{array}$ & \begin{tabular}{|c|} 
4th qtr. \\
2020
\end{tabular} & Avg. \\
\hline Japan & 40.85 & 42.78 & 50.05 & 48.26 & 45.49 & 43.38 & 36.33 & & & 39.86 \\
\hline Rotterdam & 16.73 & 16.62 & 20.21 & 19.02 & 18.15 & 14.82 & 13.18 & & & 14.00 \\
\hline China & 39.61 & 42.2 & 49.35 & 47.05 & 44.55 & 41.98 & 35.40 & & & 38.69 \\
\hline Mexico & 13.89 & 14.01 & 15.5 & 15.23 & 14.66 & 13.64 & 12.41 & & & 13.03 \\
\hline Colombia: Atlantic Ports (East) & 19.75 & 19.99 & 21.13 & 19.74 & 20.15 & 18.85 & 17.96 & & & 18.41 \\
\hline Colombia: Pacific Ports (West) & 29.38 & 29.1 & 29.02 & 32.01 & 29.88 & 27.11 & 27.55 & & & 27.33 \\
\hline \multicolumn{11}{|l|}{ Colombia: Pacific Ports (West) } \\
\hline \multicolumn{11}{|c|}{ PNW to } \\
\hline Country & \begin{tabular}{|c} 
1st qtr. \\
2019 \\
\end{tabular} & $\begin{array}{c}\text { 2nd qtr. } \\
2019 \\
\end{array}$ & $\begin{array}{c}3 r d \text { qtr. } \\
2019\end{array}$ & $\begin{array}{c}4 \text { th qtr. } \\
2019\end{array}$ & Avg. & \begin{tabular}{|c} 
1st qtr. \\
2020 \\
\end{tabular} & $\begin{array}{c}\text { 2nd qtr. } \\
2020\end{array}$ & \begin{tabular}{|c} 
3rd qtr. \\
2020 \\
\end{tabular} & \begin{tabular}{|c|}
4 th qtr. \\
2020 \\
\end{tabular} & Avg. \\
\hline Japan & 22.98 & 23.56 & 27.9 & 26.28 & 25.18 & 23.10 & 18.94 & & & 21.02 \\
\hline China & 22.44 & 22.93 & 27.28 & 25.71 & 24.59 & 22.28 & 18.20 & & & 20.24 \\
\hline
\end{tabular}

Note: qtr. = quarter; avg. = average; PNW = Pacific Northwest.

Source: O'Neil Commodity Consulting.

Figure 6. Grain vessel rates and spread, U.S. to Japan

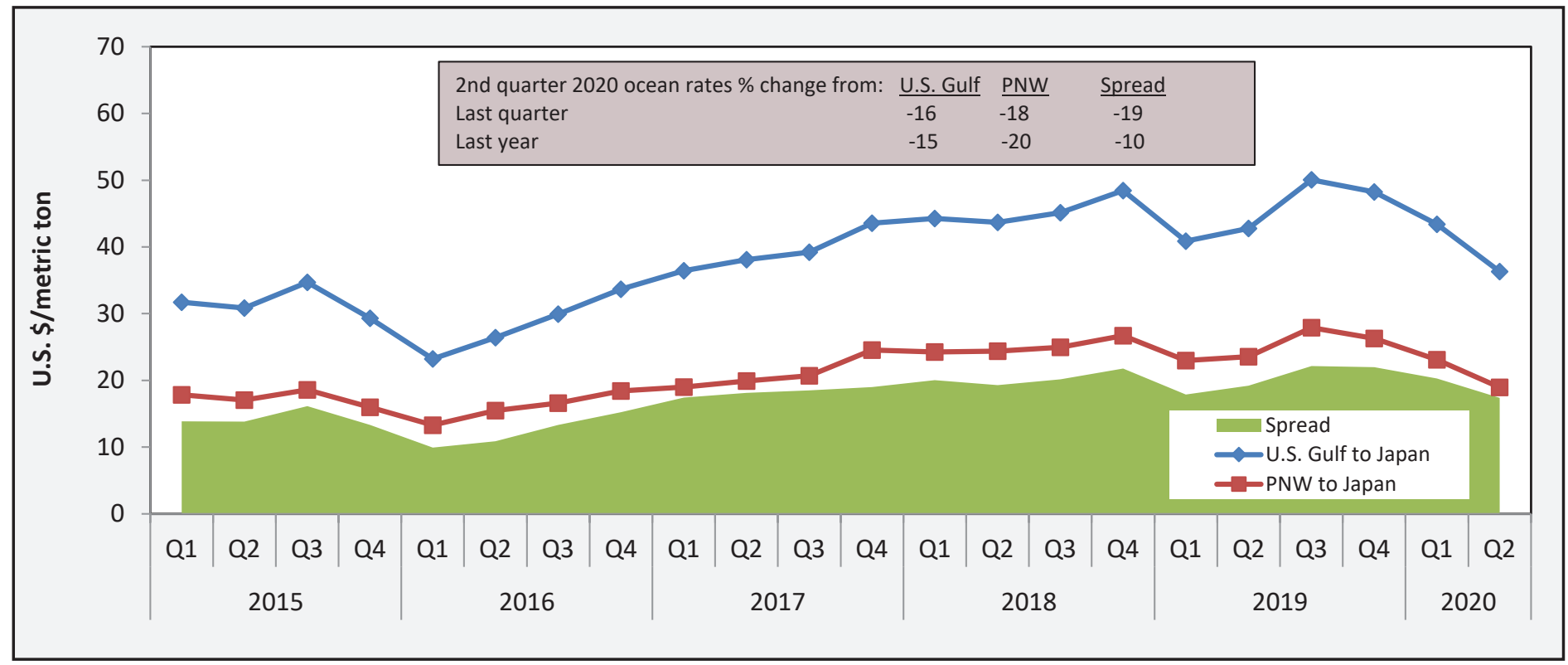

Note: $\mathrm{Q}=$ quarter; PNW = Pacific Northwest.

Source: O'Neil Commodity Consulting. 


\section{Grain Truck and Ocean Rate Advisory}

\section{CONTACTS AND LINKS}

Surajudeen Olowolayemo, Economist

E-mail: Surajudeen.Olowolayemo@usda.gov

202.720.0119

April Taylor, Economist

E-mail: April.Taylor@usda.gov

202.720.7880

Brian McGregor, Agricultural Marketing Specialist

E-mail: Brian.McGregor@usda.gov

202.720.0035

\section{Subscription Information:}

Please sign up to receive regular email announcements of the latest issue of Grain Truck and Ocean Rate Advisory by entering your email address at the following link and selecting "Transportation" under the Publications, Data, and Reports section.

https://public.govdelivery.com/accounts/USDAAMS/subscriber/new?topic id=USDAAMS 177

\section{Related Websites}

- Grain Transportation Report

- Mexico Transport Cost Indicator Report

- Brazil Soybean Transportation Indicator

- Agricultural Refrigerated Truck Quarterly

\section{Preferred Citation:}

U.S. Department of Agriculture, Agricultural Marketing Service. Grain Truck and Ocean Rate Advisory. August 2020. Web. <http://dx.doi.org/10.9752/TS265.08-2020>

Photo Credit: USDA

USDA is an equal opportunity provider, employer, and lender.

For assistance with accessibility issues related to this document, please email Jessica.Ladd@usda.gov. 